\title{
Evaluation of Promising Potato Varieties for Yield Potentiality and Late Blight Disease Tolerance in Southern Dry Zone of Karnataka, India
}

\author{
H. Amarananjundeswara*, P.S. Prasad, Soumya Shetty and G.C. Sandhya
}

AICRP on Potato, Horticultural Research and Extension Station, Hassan-573 219, Karnataka, India

*Corresponding author

\section{A B S T R A C T}

\section{Keywords}

Potato, Varieties, Growth, Yield, Late blight, Tolerance

Article Info

Accepted: 04 January 2018 Available Online: 10 February 2018
The experiment was conducted under AICRP on Potato at Horticultural Research and Extension Station, Somanahallikaval, Hassan for three consecutive years from 2014-2016 during Kharif season to know their yield potentiality and late blight disease tolerance capability of 12 different promising varieties. The varieties viz., Kufri Jyoti, Kufri Himalini, Kufri Bahar, Kufri Gaurav, Kufri Garima, Kufri Pushkar, Kufri Badshah, Kufri Khyati, Kufri Pukhraj, Kufri Surya, Kufri Ashoka and Kufri Lauvkar were evaluated under natural epiphytotic conditions. Out of twelve varieties evaluated, Kufri Himalini recorded significantly highest marketable tuber yield of $22.23 \mathrm{t} / \mathrm{ha}$ and total tuber yield of $24.17 \mathrm{t} / \mathrm{ha}$ at 90 days after sowing with least percentage of late blight incidence (3.76\%) followed by Kufri Jyoti recorded highest marketable tuber yield of $16.39 \mathrm{t} / \mathrm{ha}$ and total tuber yield of $17.95 \mathrm{t} / \mathrm{ha}$ with 5.40 per cent late blight incidence at 90 days after sowing. Therefore a variety Kufri Himalini could be recommended for commercial cultivation due to its high yield potential and late blight tolerance in Hassan district of Karnataka. Further to popularize a variety Kufri Himalini front line demonstrations were also conducted at various farmers fields of Hassan district.

\section{Introduction}

Potato (Solanum tuberosum L.) belongs to family Solanaceae is a most important crop among the vegetables. The crop finds prime position in the economy of poor and marginal farmers and plays a vital role in nutritional security by producing more food per unit area and time as compared to wheat, rice and maize in short period of time. Potato contains substantial quantity of energy, edible protein$2.8 \mathrm{~g}$, starch-16.3 g, total sugar-0.6 g, crude fibre-0.5 g, carbohydrate-22.6 $\mathrm{g}$ and vitamin-C
$25 \mathrm{mg}$ per $100 \mathrm{~g}$ fresh weight of tubers (Bhuwneshwari et al., 2013). In Hassan, it is grown during Kharif season from June-July to September-October. In India, potato is predominantly cultivated during Kharif (80\%) followed by in Rabi season (20\%).

It is grown over an area of 1.97 lakh hectares with the production of 344 lakh tones and average national yield per hectare is 21.10 tonnes in India and it is cultivated in an area of 41,000 hectares with the production of 3,61,000 tonns in Karnataka (Anon, 2015). 
Even though India stands in fourth place with respect to the production of potato in the world, but the productivity is very low as compared to other advanced countries. The possible reason could be varied agro-climatic conditions. The higher productivity could be achieved by selection of proper varieties specific to areas and other agronomical practices. As many varieties have been developed by various research institutes, universities are yet to be evaluated at different locations as the response is location specific.

Besides potato varieties show wide variation in their yielding ability when grown over a varied agro-climatic conditions. The yield and quality of potato depends upon soil, climatic conditions and variety or hybrid used. Moreover, different varieties have different soil and climatic requirements for their optimum performances. India being a vast country with varied agro-climatic regions, a single variety or hybrid may not be suitable for all the agro-climatic conditions. Hence, new varieties are need to be introduced or evaluated for specific regions.

With the objective of finding out suitable variety with higher yield potential and late blight disease tolerance for cultivation in southern Karnataka following experiment was conducted at HRES, Hassan during Kharif season.

\section{Materials and Methods}

The experiment was conducted under AICRP on Potato centre at Horticultural Research and Extension Station, Somanahallikaval, Hassan district, Karnataka state for three consecutive years from 2014-2016 to know the yield potentiality and late blight disease tolerance capability of 12 different released varieties. The released varieties viz. Kufri Jyoti, Kufri Himalini, Kufri Bahar, Kufri Gaurav, Kufri Garima, Kufri Pushkar, Kufri Badshah, Kufri
Khyati, Kufri Pukhraj, Kufri Surya, Kufri Ashoka and Kufri Lauvkar were evaluated under natural epiphytotic conditions. The experiment was laid out by using RCBD with three replications. The land was prepared for the research before sowing and FYM @ 25 t/ha was incorporated into soil. The tuber sowing was taken up during the first week of June in all three years by adopting scientific spacing of $60 \mathrm{~cm} \times 20 \mathrm{~cm}$. The recommended dosage of NPK 100:75:75 $\mathrm{kg} / \mathrm{ha}$ was incorporated. From the recommended quantity of nitrogen, 50 per cent of nitrogen applied at sowing and remaining 50 per cent of nitrogen after 30 days of sowing at earthing-up operation. The package of practices of UHS, Bagalkot was followed during different stages of crop growth and harvesting was done at 90 days after sowing. The observations related to vegetative growth attributes, yield attributes and disease scoring were recorded as follows.

\section{Vegetative growth attributes}

\section{Plant emergence (\%) at 30 days after sowing}

Total no. of tubers germinated Plant emergence $(\%)=$------------------ x 100

Total no. of tubers sown

\section{Plant height (cm)}

The maximum plant height was measured from the ground to the tip of longest leaf documented at 45 days after sowing. The mean of 5 plants in each treatment was worked out

\section{Plant spread $\left(\mathrm{cm}^{2}\right)$}

The plant spread was measured in north to south and east to west from 5 randomly selected plants at 45 days after sowing and average of plant spread was calculated in centimeter square. 


\section{Yield attributes}

\section{Marketable tuber yield (t/ha)}

Out of total tubers obtained in plant, the tubers were sorted in to 4 different grades based on their weight as small $(<25 \mathrm{~g})$, medium (26$50 \mathrm{~g})$, large $(51-75 \mathrm{~g})$ and extra-large $(>76 \mathrm{~g})$. Out of these, excluding small tubers all other grades were considered as marketable and weight was recorded and using this data marketable tuber yield per ha were calculated

\section{Total tuber yield (t/ha)}

Total tuber yield $(\mathrm{t} / \mathrm{ha})=$ Marketable tuber yield $(\mathrm{t} / \mathrm{ha})+$ small tuber yield $(<25 \mathrm{~g})$

\section{Rottage (t/ha)}

Weight of rotten tubers were recorded and using the data rottage tones per hectare was calculated

\section{Disease scoring}

Late blight incidence and late blight intensity were also computed using the following formula and Malcomson scale.

No. of Infected Plants

Late blight incidence $(\%)=$----------- x 100

Total No. of Plants

Late blight Intensity $(\%)=($ Sum of Individual Ratings / Total No. of Plants) x (100 / Max. Scale)

\section{Results and Discussion}

The observations on percentage of plant emergence, plant height, plant spread, marketable tuber yield, total tuber yield and tuber rottage were documented for three consecutive years (2014-16). All varieties shown significant difference over their growth performance, yield potentiality and disease tolerance capability.

\section{Vegetative growth attributes}

The pooled data analysis for growth parameters indicated that, among all the varieties Kufri Pushkar registered highest percentage of plant emergence of about 89.08 at 30 days after sowing (Table 1), highest plant height of $71.26 \mathrm{~cm}$ was noticed in Kufri Garima and highest plant spread of $51.67 \mathrm{~cm}^{2}$ was registered in Kufri Khyati at 45 days after sowing (Table 1). The better performance of these varieties might be due to its genetic make-up and its better adoptability to prevailing environmental conditions (Gobana, 2002).

Yield attributes and late blight incidence percentage

The pooled data analysis for tuber yield indicated that, among twelve varieties evaluated Kufri Himalini registered significantly highest marketable tuber yield of $22.23 \mathrm{t} / \mathrm{ha}$ (Table 2) and total tuber yield of $24.17 \mathrm{t} / \mathrm{ha}$ at 90 days after sowing with least percentage of late blight incidence $(3.76 \%)$ (Table 3) followed by Kufri Jyoti recorded good marketable tuber yield of $16.39 \mathrm{t} / \mathrm{ha}$ and total tuber yield of $17.95 \mathrm{t} / \mathrm{ha}$ with moderate tolerance to late blight disease $(5.40 \%)$. However, least rottage of $0.39 \mathrm{t} / \mathrm{h}$ a tubers were noticed in Kufri Himalini followed by Kufri Surya $(0.50 \mathrm{t} / \mathrm{ha})$. The results are in agreement with other researchers who investigated that marketable tuber yield was significantly varied by variety, location and genotypes $\mathrm{x}$ environment interaction (Elfinesh, 2008, Gebreselassie et al., 2016, Pandey et al., 2004 and Kumar et al., 2007). The results obtained demonstrated that genetic yield differences are not caused by one single growth or development parameter (Meyling et al., 1971) (Fig. 1 and 2). 
Table.1 Performance of promising potato varieties on different growth parameters during Kharif season

\begin{tabular}{|c|c|c|c|c|c|c|c|c|c|c|c|c|}
\hline \multirow[t]{2}{*}{ Variety } & \multicolumn{4}{|c|}{ Plant Emergence (\%) at 30 DAS } & \multicolumn{4}{|c|}{ Plant Height (cm) at 45 DAS } & \multicolumn{4}{|c|}{ Plant Spread $\left(\mathrm{cm}^{2}\right)$ at 45 DAS } \\
\hline & 2014 & 2015 & 2016 & Pooled & 2014 & 2015 & 2016 & Pooled & 2014 & 2015 & 2016 & Pooled \\
\hline Kufri Himalini & 74.00 & 90.00 & 82.46 & 82.15 & 40.00 & 55.00 & 65.25 & 53.42 & 43.75 & 52.00 & 54.13 & 49.96 \\
\hline Kufri Jyoti & 78.00 & 91.70 & 86.28 & 85.33 & 51.50 & 57.00 & 53.1 & 53.87 & 44.75 & 53.25 & 45.25 & 47.75 \\
\hline Kufri Surya & 97.00 & 81.29 & 86.58 & 88.29 & 48.00 & 51.50 & 61.9 & 53.80 & 52.50 & 43.50 & 49.75 & 48.58 \\
\hline Kufri Pukhraj & 94.00 & 87.19 & 85.73 & 88.97 & 65.50 & 71.50 & 62.45 & 66.48 & 45.00 & 47.00 & 51.50 & 47.83 \\
\hline Kufri Pushkar & 97.00 & 86.05 & 84.19 & 89.08 & 70.00 & 79.50 & 63.05 & 70.85 & 42.75 & 50.75 & 51.63 & 48.38 \\
\hline Kufri Bahar & 81.00 & 84.66 & 85.44 & 83.70 & 42.50 & 59.50 & 55.6 & 52.53 & 32.25 & 50.25 & 44.38 & 42.29 \\
\hline Kufri Khyati & 97.00 & 83.08 & 81.78 & 87.29 & 67.50 & 71.00 & 70.25 & 69.58 & 48.50 & 47.25 & 59.25 & 51.67 \\
\hline Kufri Gaurav & 79.00 & 84.92 & 84.68 & 82.87 & 49.50 & 54.00 & 49.5 & 51.00 & 43.50 & 48.50 & 56.63 & 49.54 \\
\hline Kufri Badshah & 82.00 & 80.28 & 83.38 & 81.89 & 66.00 & 73.50 & 63.7 & 67.73 & 41.75 & 53.75 & 45.50 & 47.00 \\
\hline Kufri Ashoka & 94.00 & 76.93 & 80.11 & 83.68 & 68.00 & 74.00 & 71.45 & 71.15 & 54.25 & 46.00 & 44.19 & 48.15 \\
\hline Kufri Garima & 92.00 & 88.52 & 86.42 & 88.98 & 67.00 & 76.00 & 70.78 & 71.26 & 38.75 & 55.25 & 51.00 & 48.33 \\
\hline Kufri Lauvkar & 78.00 & 81.46 & 83.5 & 80.99 & 40 & 47.15 & 51.24 & 46.13 & 48.25 & 52.26 & 51.50 & 50.67 \\
\hline S Em \pm & 2.57 & 5.36 & 1.33 & 3.51 & 1.68 & 6.47 & 2.33 & 3.00 & 4.16 & 1.63 & 3.45 & 3.03 \\
\hline $\mathrm{CD}(\mathrm{p}=0.05)$ & 8.00 & 12.14 & 3.86 & 10.29 & 5.23 & 14.40 & 6.77 & 8.80 & 12.20 & 4.77 & 10.00 & 8.88 \\
\hline $\mathrm{CV}$ & 4.18 & 6.40 & 3.16 & 7.13 & 5.13 & 9.53 & 7.57 & 8.57 & 16.13 & 5.63 & 13.72 & 10.85 \\
\hline
\end{tabular}


Table.2 Performance of promising potato varieties on different yield parameters during Kharif season

\begin{tabular}{|c|c|c|c|c|c|c|c|c|c|c|c|c|}
\hline \multirow[t]{2}{*}{ Variety } & \multicolumn{4}{|c|}{ Marketable tuber yield (t/ha) at 90 DAS } & \multicolumn{4}{|c|}{ Total tuber yield (t/ha) at 90 DAS } & \multicolumn{4}{|c|}{ Rottage (t/ha) at 90 DAS } \\
\hline & 2014 & 2015 & 2016 & Pooled & 2014 & 2015 & 2016 & Pooled & 2014 & 2015 & 2016 & Pooled \\
\hline Kufri Himalini & 28.23 & 17.44 & 21.02 & 22.23 & 30.68 & 19.69 & 22.14 & 24.17 & 0.20 & 0.40 & 0.56 & 0.39 \\
\hline Kufri Jyoti & 14.07 & 14.50 & 20.60 & 16.39 & 15.70 & 16.60 & 21.56 & 17.95 & 1.00 & 1.24 & 0.39 & 0.88 \\
\hline Kufri Surya & 13.62 & 9.83 & 16.78 & 13.41 & 15.12 & 11.00 & 19.19 & 15.10 & 0.40 & 0.60 & 0.49 & 0.50 \\
\hline Kufri Pukhraj & 17.52 & 11.70 & 18.28 & 15.83 & 19.26 & 13.80 & 20.59 & 17.88 & 0.86 & 1.08 & 0.54 & 0.83 \\
\hline Kufri Pushkar & 20.24 & 11.50 & 15.59 & 15.78 & 21.88 & 13.50 & 18.13 & 17.84 & 0.68 & 0.94 & 0.36 & 0.66 \\
\hline Kufri Bahar & 15.86 & 13.50 & 11.80 & 13.72 & 17.44 & 14.90 & 13.61 & 15.32 & 0.98 & 1.35 & 0.29 & 0.87 \\
\hline Kufri Khyati & 15.06 & 7.40 & 10.22 & 10.89 & 16.54 & 9.48 & 12.53 & 12.85 & 0.87 & 1.26 & 0.18 & 0.77 \\
\hline Kufri Gaurav & 8.08 & 5.17 & 12.58 & 8.61 & 9.25 & 7.950 & 14.56 & 10.59 & 0.30 & 0.40 & 1.26 & 0.65 \\
\hline Kufri Badshah & 21.77 & 10.40 & 14.60 & 15.59 & 24.29 & 12.50 & 16.67 & 17.82 & 0.90 & 1.18 & 0.27 & 0.78 \\
\hline Kufri Ashoka & 18.99 & 12.60 & 12.00 & 14.53 & 20.66 & 14.70 & 14.02 & 16.46 & 1.27 & 1.06 & 2.00 & 1.44 \\
\hline Kufri Garima & 12.60 & 6.28 & 11.00 & 9.96 & 14.14 & 9.06 & 11.50 & 11.57 & 0.37 & 0.65 & 0.50 & 0.51 \\
\hline Kufri Lauvkar & 14.18 & 11.84 & 12.00 & 12.67 & 14.77 & 13.77 & 15.00 & 14.85 & 1.40 & 1.00 & 2.50 & 1.63 \\
\hline $\mathrm{S} \mathrm{Em+}$ & 0.62 & 0.67 & 1.14 & 1.70 & 1.43 & 0.72 & 0.88 & 1.67 & 0.09 & 0.07 & 0.04 & 0.26 \\
\hline $\mathrm{CD}(\mathrm{p}=0.05)$ & 1.94 & 2.15 & 3.30 & 4.97 & 4.44 & 2.30 & 2.55 & 4.90 & 0.29 & 0.23 & 0.13 & 0.77 \\
\hline $\mathrm{CV}$ & 3.19 & 12.80 & 14.81 & 19.37 & 6.69 & 11.40 & 10.15 & 18.05 & 17.28 & 14.61 & 14.88 & 55.61 \\
\hline
\end{tabular}

DAS: Days After Sowing

Table.3 Performance of promising potato varieties against late blight disease tolerance during Kharif season

\begin{tabular}{|c|c|c|c|c|}
\hline Variety & Late Blight incidence (\%) & Late Blight Intensity & Leaf Spot Disease (\%) & Viral Disease (\%) \\
\hline Kufri Jyoti & 5.40 & 8 & - & 5.25 \\
\hline Kufri Bahar & 13.68 & 8 & - & 6.12 \\
\hline Kufri Gaurav & 20.42 & 9 & - & 5.65 \\
\hline Kufri Himalini & 3.76 & 9 & - & 4.12 \\
\hline Kufri Pushkar & 23.54 & 9 & - & 5.45 \\
\hline Kufri Badshah & 11.17 & 8 & - & 8.50 \\
\hline Kufri Khyati & 25.21 & 8 & - & 7.50 \\
\hline Kufri Pukhraj & 11.11 & 9 & - & 15.17 \\
\hline Kufri Surya & 4.69 & 9 & - & 6.25 \\
\hline Kufri Ashoka & 31.96 & 9 & - & 20.16 \\
\hline Kufri Garima & 18.00 & 9 & - & 3.00 \\
\hline Kufri Lauvkar & 21.35 & 9 & & 6.23 \\
\hline
\end{tabular}


Table.4 Front Line Demonstration (FLD) on promising varieties conducted at Hassan District, Karnataka state during 2015-16

\begin{tabular}{|c|c|c|c|c|c|}
\hline \multirow{2}{*}{$\begin{array}{l}\text { S. } \\
\text { No. }\end{array}$} & \multirow[t]{2}{*}{ Name of the farmer } & \multirow[t]{2}{*}{ Locations } & \multicolumn{3}{|c|}{ Tuber yield (t/ha) } \\
\hline & & & $\begin{array}{l}\text { Kufri } \\
\text { Surya }\end{array}$ & $\begin{array}{l}\text { Kufri } \\
\text { Jyoti }\end{array}$ & $\begin{array}{l}\text { Kufri } \\
\text { Himalini }\end{array}$ \\
\hline 1 & Mr. Thimmegowda & $\begin{array}{c}\text { Bageshapura (Village) } \\
\text { Arasikere (Taluk) } \\
\text { Hassan (District) }\end{array}$ & 19.17 & 18.70 & 26.40 \\
\hline 2 & Mr. Devegowda & $\begin{array}{c}\text { Marenhalli (Village) } \\
\text { Shantigrama (Hobli) } \\
\text { Hassan (Taluk) } \\
\text { Hassan (District) }\end{array}$ & 6.90 & 14.60 & 19.70 \\
\hline 3 & Mr. Shankarlingegowda & $\begin{array}{c}\text { Sopnahalli (Village) } \\
\text { Hassan (Taluk) } \\
\text { Hassan (District) }\end{array}$ & 11.25 & 8.00 & 23.90 \\
\hline 4 & Mr. Ningegowda & $\begin{array}{c}\text { Hullangane (Village) } \\
\text { Arakalgudu (Taluk) } \\
\text { Hassan (District) }\end{array}$ & 20.83 & 25.83 & 25.83 \\
\hline 5 & Mr. Krishnegowda & $\begin{array}{l}\text { Hulikallu (Village) } \\
\text { Arakalgudu (Taluk) } \\
\text { Hassan (District) }\end{array}$ & 15.00 & 14.41 & 19.16 \\
\hline 6 & Mr. Laxmegowda & $\begin{array}{c}\text { Annenahalli (Village) } \\
\text { Channarayapattana } \\
\text { (Taluk) } \\
\text { Hassan (District) }\end{array}$ & 17.49 & 20.67 & 28.62 \\
\hline 7 & Mr. Eshwaregowda & $\begin{array}{c}\text { Umadevarhalli (Village) } \\
\text { Alur (Taluk) } \\
\text { Hassan (District) }\end{array}$ & 14.60 & 16.20 & 23.17 \\
\hline & & Average yield (t/ha) & 15.03 & 16.92 & 23.83 \\
\hline
\end{tabular}

\section{Malcomson scale}

\begin{tabular}{|c|c|}
\hline Per cent Area Infected (\%) & Score \\
\hline Trace of infection & 9 \\
\hline 10 & 8 \\
\hline $11-25$ & 7 \\
\hline $26-40$ & 6 \\
\hline $41-60$ & 5 \\
\hline $61-70$ & 4 \\
\hline $71-80$ & 3 \\
\hline $81-90$ & 2 \\
\hline Collapsed & 1 \\
\hline
\end{tabular}




\section{Best performing potato varieties}

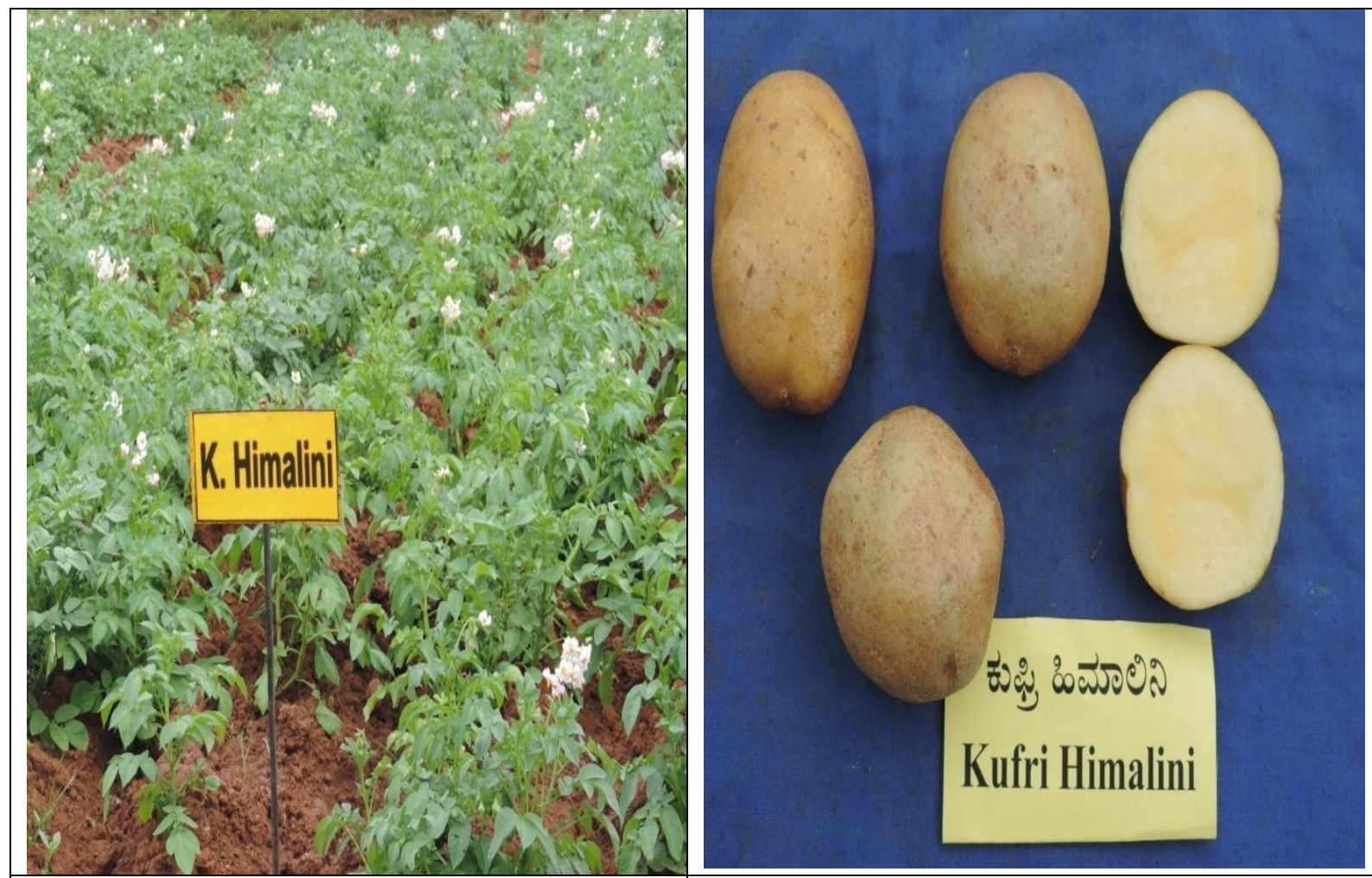

Plate.1 Crop view of variety Kufri Himalini

Plate.2 Kufri Himalini tubers

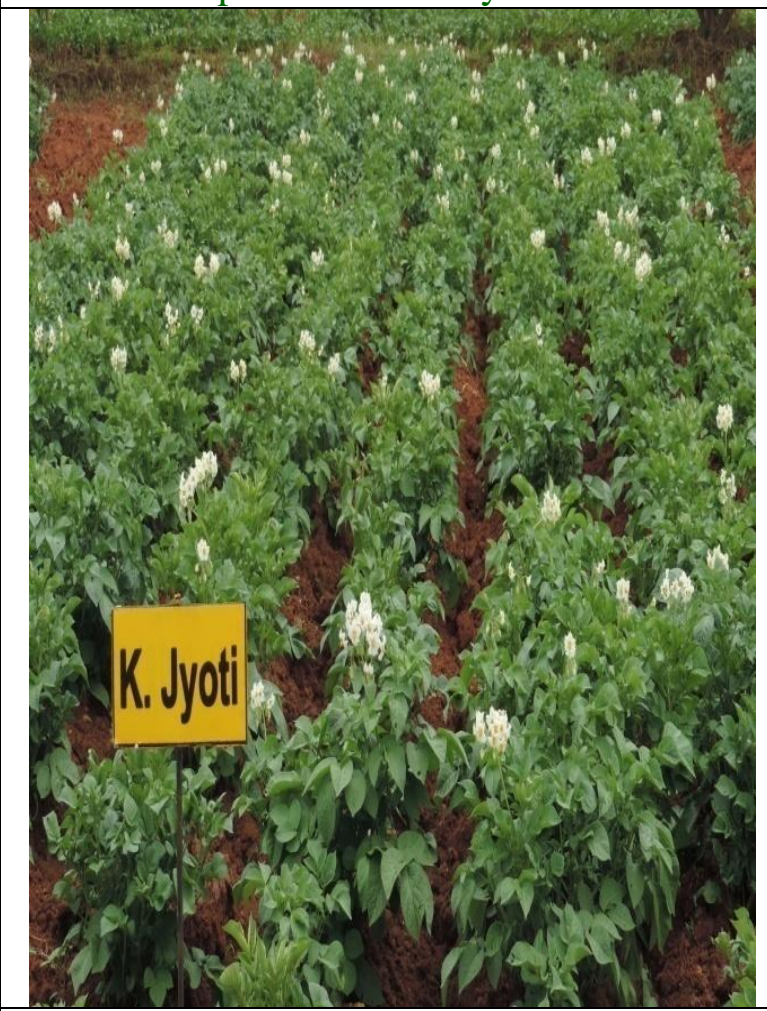

Plate.3 Crop view of variety Kufri Jyoti

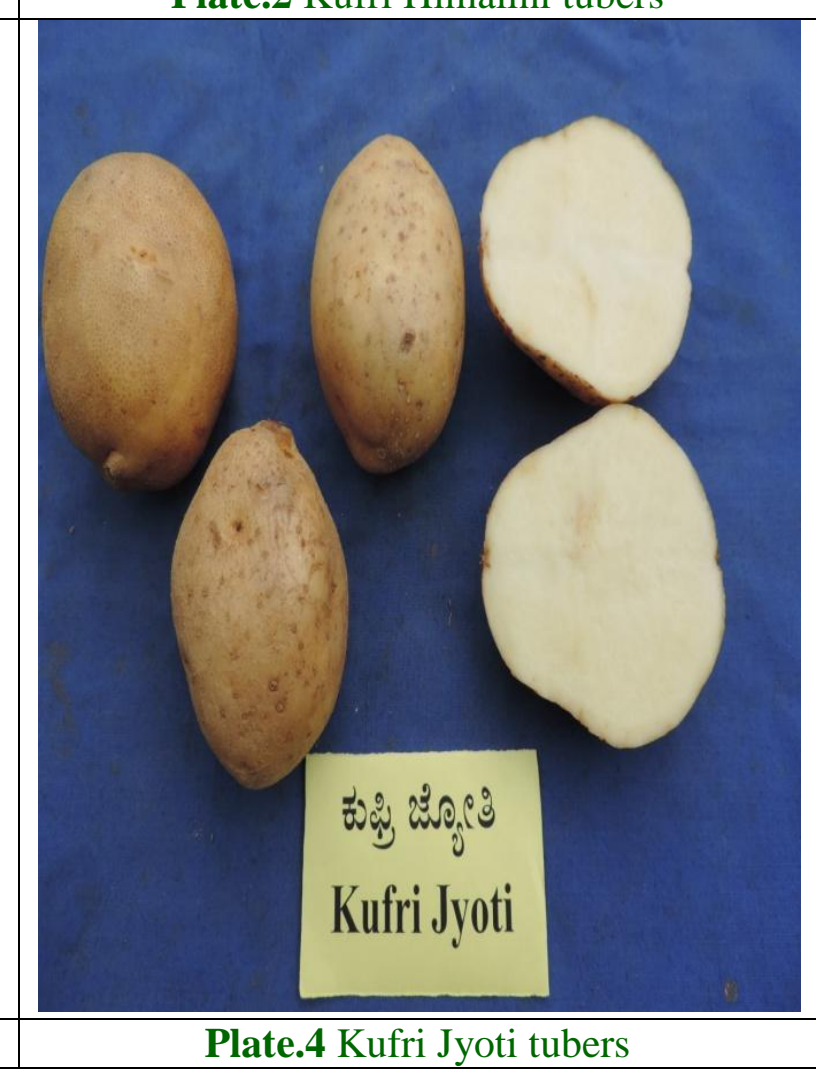


Fig.1 Marketable and total tuber yield (t/ha) of different potato varieties as influenced by Kharif season

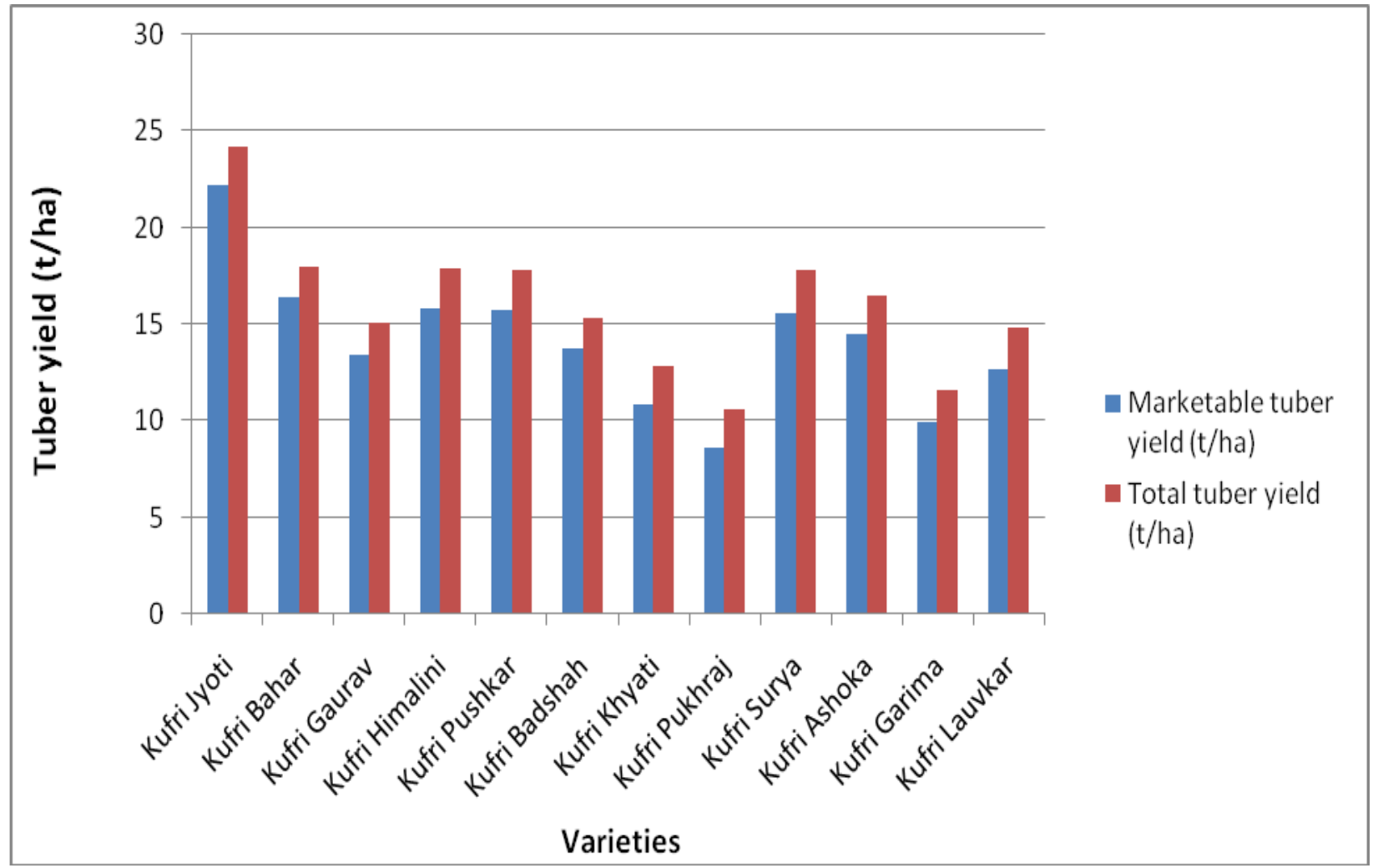

Fig.2 Late blight incidence (\%) in different potato varieties as influenced by Kharif season

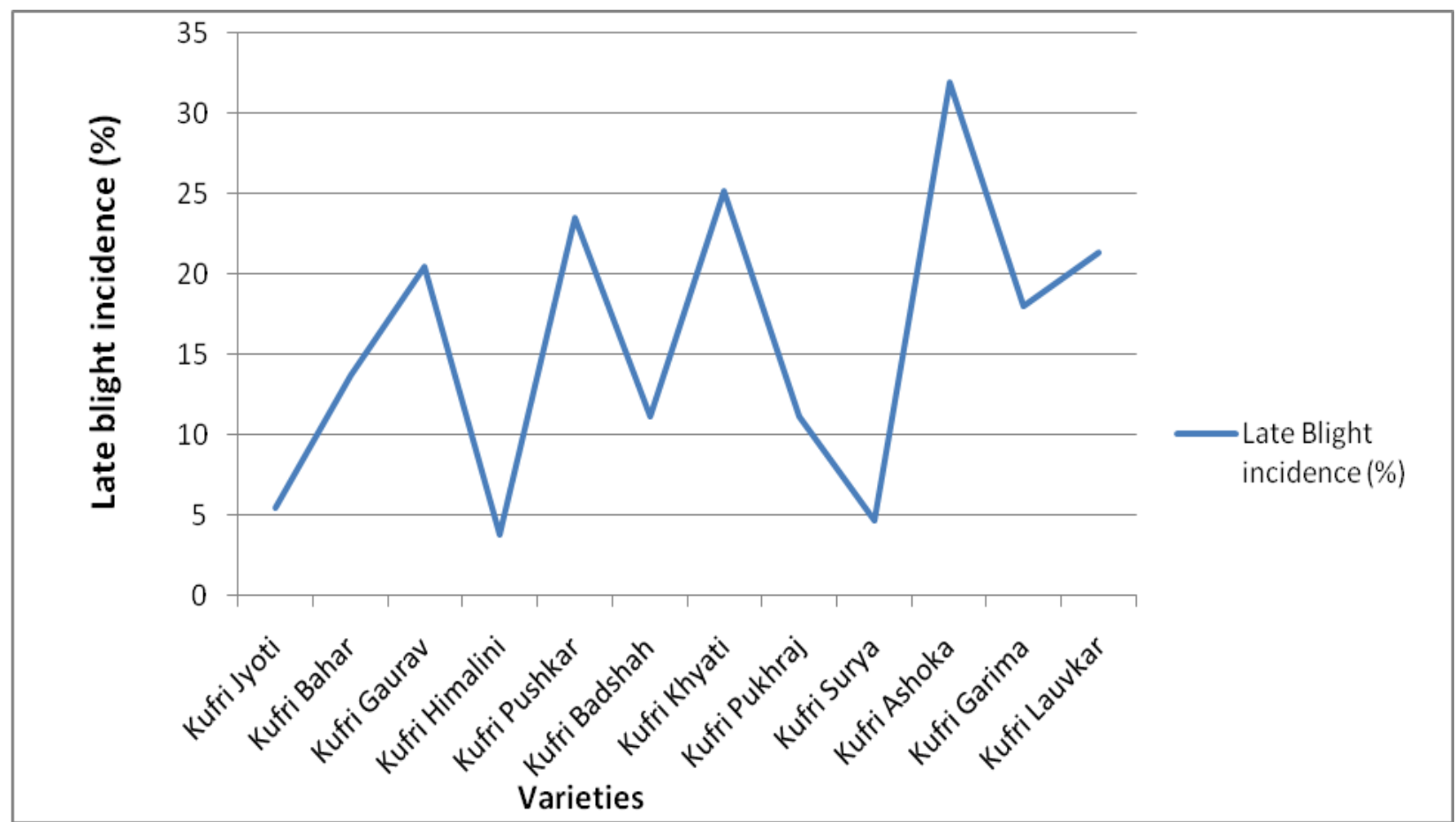




\section{Front Line Demonstration (FLD)}

Front line demonstrations were conducted at various farmers' fields in different talukas of Hassan district of Karnataka state with best three varieties of the region viz., Kufri Himalini, Kufri Surya and Kufri Jyoti. A variety Kufri Himalini was documented highest tuber yield (average yield- $23.83 \mathrm{t} / \mathrm{ha}$ ) in all farmers field over other varieties and found highly tolerant to late blight disease (Table 3 and 4).

Therefore, it is evident from these results that Kufri Himalini is a high yielding, late blight resistant variety than other cultivars. The cultivation of this variety will replace the other varieties in combating late blight disease more effectively as these carry resistance genes from different sources.

In addition, it has a high level of resistance to late blight in foliage and moderate resistance in the tubers was reported by Joseph et al., 2007.

The area and production of potato in Karnataka especially in Hassan district has been reduced drastically since 2005 due to severe incidence of late blight disease. This is mainly due to occurrence of congenial weather condition for development of late blight during cropping season and susceptibility showed by Kufri Jyoti variety to late blight disease.

The results of experiment conducted at HRES, Hassan over three consecutive years revealed that, a variety Kufri Himalini is found best suitable variety having higher yield potentiality and late blight disease tolerance over other released promising varieties evaluated at this centre. Therefore, this variety could be recommended for commercial cultivation in Southern Dry Zone of Karnataka.

\section{Acknowledgment}

Central Potato Research Institute, Shimla, Himachal Pradesh

Central Potato Research Station, Modipuram, Uattar Pradesh for supplying seed tubers

Department of Horticulture, Hassan, Karnataka for their co-operation in conducting front line demonstrations.

\section{References}

Anonymous, 2015. Horticulture Database, National Horticulture Board, Bengaluru, Karnataka

Bhuwneshwari, Satish, Verma, K., Kamal Narayan and Paikra, M.S., 2013. Evaluation of processing potato genotypes for growth, yield and yield attributes under Chhattisgarh condition. The Asian J. Hort., 8 (1): 241-245.

Elfinesh, F., 2008. Processing quality of improved potato (Solanum tuberosum L.) varieties as influenced by growing environment, genotype and blanching. M. Sc. Thesis, School of Graduate Studies of Haramaya University, Ethiopia

Gebreselassie, H., Mohamed, W and Shimelis, B., 2016. Evaluation of potato (Solanum tuberosum L.) varieties for yield and yield components in Eastern Ethiopia, J. Bio. Agri. and Healthcare, 6(5):146-154.

Gobana, D. R., 2002. Genetic variability, heritability and path co-efficient studies in Potato (Solanum tuberosum L.). M.Sc (Hort.) Thesis, Univ. Agric. Sci. Dharwad, Karnataka (India). p.166

Joseph, T. A., Singh, B. P., Kaushik, S. K., Bhardwaj, V., Pandey, S. K., Singh, S. V., Gopal, J., Singh, P. H and Gupta, V. K., 2007. Kufri Himalini: a high yielding, late blight resistant potato 
variety suitable for cultivation in Indian hills, Potato J., 34 (3 - 4): 153-158.

Kumar, S., Khade, H.D., Dhokane, V.S., Bethere, A.G and Sharma A., 2007. Irradiation in combination with higher storage temperatures maintains chipmaking quality of potato. J. Food Sci., 72 (6): 402-406.

Meyling, H. D and Bodlaender, K.B., 1981. Varietal differences in growth, development and tuber production of potatoes. Neth. J. Agric. Sci., 29: 113127.

Pandey, S.K., Singh, S.V., Kumar, P and Manivel, P., 2004. Sustaining potato chipping Industry from western and central Uttar Pradesh: Adoption of suitable varieties. Potato J., 31(3-4): 119-127.

\section{How to cite this article:}

Amarananjundeswara, H., P.S. Prasad, Soumya Shetty and Sandhya, G.C. 2018. Evaluation of Promising Potato Varieties for Yield Potentiality and Late Blight Disease Tolerance in Southern Dry Zone of Karnataka, India. Int.J.Curr.Microbiol.App.Sci. 7(02): 341-350. doi: https://doi.org/10.20546/ijcmas.2018.702.045 